Mathematical Modelling and Analysis

Volume 10 Number 1, 2005, PAges 41-50

(C) 2005 Technika ISSN 1392-6292

\title{
QUASILINEARIZATION AND MULTIPLE SOLUTIONS OF THE EMDEN-FOWLER TYPE EQUATION
}

\author{
I. YERMACHENKO ${ }^{1}$ and F. SADYRBAEV ${ }^{2}$ \\ ${ }^{1}$ Daugavpils University \\ Parades str. 1, Daugavpils, Latvia \\ E-mail: inari@dau. $1 \mathrm{v}$ \\ ${ }^{2}$ Institute of Mathematics and Computer Science, University of Latvia \\ Rainis bl. 29, Riga, Latvia \\ E-mail: felix@cclu. lv
}

Received June 29, 2004; revised November 17, 2004

\begin{abstract}
Existence and multiplicity of solutions of the problem $x^{\prime \prime}=-q(t)|x|^{p}$ sign $x$ (i), $x(0)=x(1)=0 \quad$ (ii) are investigated by reducing equation (i) to a quasi-linear one so that both equations are equivalent in some domain $\Omega$. If a solution of corresponding quasilinear problem is located in the domain of equivalence $\Omega$, then this solution solves the original problem also. If this process of quasilinearization is possible for multiple essentially different linear parts, then multiple solutions to the problem $(i),($ ii $)$ exist.
\end{abstract}

Key words: quasi-linear equation, $i$-nonresonant linear part, $i$-type solution, quasi-linearization

\section{Introduction}

It is well known that the boundary value problem

$$
\left\{\begin{array}{l}
x^{\prime \prime}=f(t, x), \quad t \in I:=[0,1], \quad f \in C(I \times R, R), \\
x(0)=0, \quad x(1)=0
\end{array}\right.
$$

is solvable if $f$ is bounded, that is, there exists $M \in(0,+\infty)$ such that $|f|<M$ for any values of arguments.

If $f$ is not bounded, the existence of a solution is not guaranteed. In some cases boundary value problems with unbounded $f$ can be reduced to a problem

$$
x^{\prime \prime}=F(t, x), \quad x(0)=x(1)=0,
$$


where $F$ is continuous and bounded. This is the case, for instance, if there exist the so called upper and lower functions $\beta$ and $\alpha$, which satisfy the conditions

$$
\begin{aligned}
& \alpha<\beta, \quad \alpha^{\prime \prime} \geq f(t, \alpha), \quad \beta^{\prime \prime} \leq f(t, \beta), \quad \forall t \in I, \\
& \alpha(0) \leq 0 \leq \beta(0), \quad \alpha(1) \leq 0 \leq \beta(1) .
\end{aligned}
$$

A continuous and bounded right side $F$ in (1.2) can be constructed in such a way that $F$ and $f$ coincide on the set $\{(t, x): 0 \leq t \leq 1, \alpha \leq x \leq \beta\}$ and any solution $x(t)$ of the problem (1.2) satisfies the estimates $\alpha \leq x \leq \beta$. Obviously $x$ solves the problem (1.1) also. The interested reader may consult the book [1] to learn more about the upper and lower functions.

We are motivated by the works of Erbe [2] and Jackson and Schrader [3]. They showed that if the upper and lower functions exist then more can be said about solutions of the boundary value problem. Their result, when adapted to the problem (1.1), says that at least one solution $\xi$ to the problem exists such that the respective equation of variations

$$
y^{\prime \prime}=f_{x}(t, \xi(t)) y
$$

is disconjugate on the interval $I$, that is, a solution $y$ of the Cauchy problem (1.3), $y(0)=0, \quad y^{\prime}(0)=1$ does not vanish in the interval $(0,1)$ (it may vanish at $t=1$, however).

This result is valid for problems of the type (1.1) with bounded right sides since then $\alpha$ and $\beta$ can be constructed as appropriate quadratic functions $(\beta=-M(t-$ $\left.\frac{1}{2}\right)^{2}+M, \alpha=M\left(t-\frac{1}{2}\right)^{2}-M$, for example).

It is known as well ([1]) that the quasi-linear problem ( $F_{1}$ is bounded)

$$
\left(L_{2} x\right)(t):=x^{\prime \prime}+P(t) x^{\prime}+Q(t)=F_{1}(t, x), \quad x(0)=x(1)=0
$$

with nonresonant linear part $\left(L_{2} x\right)(t)$ (see Definition 1 below) is solvable. It was shown in [4] that the quasi-linear problem (1.4) with oscillatory linear part $\left(L_{2} x\right)(t)$ has a solution $\xi(t)$ such that the respective equation of variations has similar oscillatory properties. The important conclusion of the result in [4] is that if the original problem of the type (1.1) can be reduced to various quasi-linear problems with essentially different oscillatory linear parts, then the original problem has multiple solutions.

The intent of this paper is to apply the idea above and the main result in [4] to prove that the Emden - Fowler equation

$$
x^{\prime \prime}=-q(t)|x|^{p} i \operatorname{sign} x
$$

has multiple solutions (of different types) subject to the boundary conditions (1.1).

Precise definitions and statements of the respective results are given in Section 2. Section 3 is devoted to investigation of the Dirichlet boundary value problem for the second order Emden - Fowler equation provided that the coefficient $q(t)$ is positive. It is shown that for $p \sim 1$ there exist multiple solutions (of different types) of the problem under consideration. 


\section{Quasilinearization and Multiple Solutions}

Several definitions will be used in the sequel.

DEFinition 1 . The linear part $\left(L_{2} x\right)(t)$ is called by a nonresonant (with respect to the boundary conditions), if the homogeneous problem

$$
\left(L_{2} x\right)(t)=0, \quad x(0)=0, \quad x(1)=0
$$

has only the trivial solution.

For instance, a linear part $\left(L_{2} x\right)(t):=x^{\prime \prime}+k^{2} x$ is nonresonant, if $k \neq \pi i$, $i=0,1, \ldots$, that is, the coefficient $k$ belongs to one of the intervals

$$
(0, \pi),(\pi, 2 \pi), \ldots,(i \pi,(i+1) \pi), \ldots
$$

Definition 2. The linear part $\left(L_{2} x\right)(t)$ is $i$-nonresonant in the interval $[0,1]$ with respect to the Dirichlet boundary conditions in (1.1), if a solution of the Cauchy problem

$$
\left(L_{2} x\right)(t)=0, \quad x(0)=0, \quad x^{\prime}(0)=1
$$

has exactly $i$ zeros in the interval $(0,1)$ and $x(1) \neq 0$.

For instance, a linear part $\left(L_{2} x\right)(t):=x^{\prime \prime}+k^{2} x$ is $i$-nonresonant for any $k$ of the form $k=\frac{\pi}{2}+\pi i, i=0,1,2, \ldots$

Definition 3. We will say that $\xi(t)$ is an $i$-type solution of the second order boundary value problem

$$
\left(L_{2} x\right)(t)=f(t, x), \quad x(0)=0, \quad x(1)=0,
$$

if for small enough $\alpha>0$ the difference $u(t ; \alpha)=x(t ; \alpha)-\xi(t)$ has exactly $i$ zeros in the interval $(0,1)$ and $u(1 ; \alpha) \neq 0$, where $x(t ; \alpha)$ is a solution of the equation

$$
\left(L_{2} x\right)(t)=f(t, x),
$$

which satisfies the initial conditions

$$
x(0 ; \alpha)=\xi(0)=0, \quad x^{\prime}(0 ; \alpha)=\xi^{\prime}(0)+\alpha .
$$

The following theorem is valid ([4, Th. 2.1]).

Theorem 1. Quasi-linear problem (2.3) with an $i$-nonresonant linear part $\left(L_{2} x\right)(t)$ has an i-type solution.

DEFinition 4. Let the equation (1.1) and quasi-linear equation (2.4) be equivalent in a domain

$$
\Omega_{N}=\{(t, x): 0 \leq t \leq 1,|x|<N\} .
$$

Suppose that any solution $x(t)$ of the quasi-linear problem (2.4) with boundary conditions in (1.1) satisfies the estimate

$$
|x(t)|<N, \quad \forall t \in[0,1] .
$$

We will say then that the problem (1.1) allows for quasilinearization with respect to a domain $\Omega_{N}$ and a linear part $\left(L_{2} x\right)(t)$. 
The statements below follow from Theorem 1 .

Corollary 1. If the problem (1.1) allows for quasilinearization with respect to some domain $\Omega_{N}$ and some $i$-nonresonant linear part $\left(L_{2} x\right)(t)$, then it has an $i$-type solution.

Corollary 2. Suppose that the problem (1.1) allows for quasilinearization with respect to $n$ domains of the form (2.6) and $n$ essentially different (in the sense of Definition 2) linear parts. Then it has at least $n$ different solutions.

Analogous results on multiple solutions are obtained for the second order Neumann [6] and Sturm-Liouville [5] boundary value problems.

\section{The Emden - Fowler Equation}

Consider the boundary value problem

$$
\begin{aligned}
& x^{\prime \prime}=-q(t)|x|^{p} \operatorname{sign} x, \quad p>0, \quad q \in C(I,(0,+\infty)), \\
& x(0)=0, \quad x(1)=0 .
\end{aligned}
$$

Theorem 2. Suppose that

$$
0<q_{1} \leq q(t) \leq q_{2}
$$

and the inequality

$$
\frac{k}{|\sin k|}<\beta \frac{p^{\frac{p}{p-1}}}{|p-1|}\left(\frac{q_{1}}{q_{2}}\right)^{\frac{1}{|p-1|}}
$$

holds for some $k \in(i \pi,(i+1) \pi),(i=0,1, \ldots)$, where $\beta>1$ is the root of the equation

$$
\beta^{p}=\beta+(p-1) p^{\frac{p}{1-p}} .
$$

Then there exists an i-type solution of the problem (3.1), (3.2).

Proof. Let us consider instead of the equation (3.1) the equivalent one

$$
x^{\prime \prime}+k^{2} x=k^{2} x-q(t)|x|^{p} \operatorname{sign} x .
$$

The linear part $\left(L_{2} x\right)(t):=x^{\prime \prime}+k^{2} x$ is nonresonant with respect to the boundary conditions (3.2). We wish to make the right side in (3.6) bounded. Denote

$$
f_{k}(t, x):=k^{2} x-q(t)|x|^{p} \operatorname{sign} x .
$$

The function $f_{k}(t, x)$ is odd in $x$ for fixed $t$. Let us consider it for nonnegative values of $x$. There exists a positive point of local extremum $x_{0}$ (it is either a point of maximum in case of $p>1$ or a point of minimum in case of $0<p<1$ )

$$
x_{0}=\left(\frac{k^{2}}{p q(t)}\right)^{\frac{1}{p-1}} .
$$




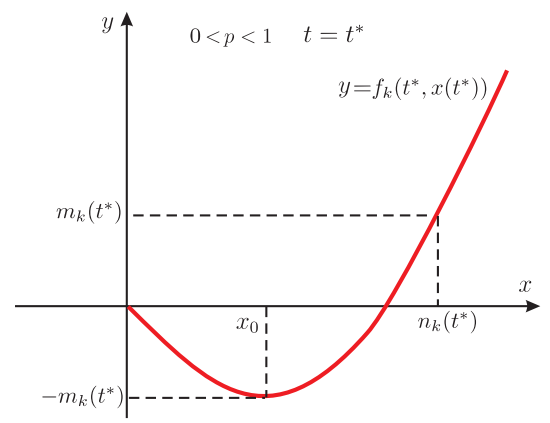

Figure 1. Existence of a number $n_{k}$.

Figure 1 illustrates the case of $0<p<1$ for a fixed $t=t^{*}$.

We can calculate the value of the function at the point of minimum $x_{0}$. Set

$$
m_{k}(t)=\left|f_{k}\left(t, x_{0}\right)\right|=\left(\frac{k^{2}}{p}\right)^{\frac{p}{p-1}}|p-1| q(t)^{\frac{1}{1-p}} .
$$

Choose $n_{k}(t)$ such that

$$
|x| \leq n_{k}(t) \Rightarrow\left|f_{k}(t, x)\right| \leq m_{k}(t), \quad \forall t \in I .
$$

The value of $n_{k}(t)$ is computed by solving the equation

$$
f_{k}(t, x)=-f_{k}\left(t, x_{0}\right)
$$

or, equivalently, that of

$$
k^{2} x-q(t) x^{p}=\left(\frac{k^{2}}{p}\right)^{\frac{p}{p-1}}(1-p) q(t)^{\frac{1}{1-p}}
$$

with respect to $x$ for any fixed $t$. Computation gives that

$$
n_{k}(t)=\left(\frac{k^{2}}{q(t)}\right)^{\frac{1}{p-1}} \beta,
$$

where a constant $\beta>1$ is described in (3.5). Set

$$
N_{k}=\min \left\{n_{k}(t): t \in[0,1]\right\}, \quad M_{k}=\max \left\{m_{k}(t): t \in[0,1]\right\} .
$$

Let us consider the quasi-linear equation

$$
x^{\prime \prime}+k^{2} x=F_{k}(t, x),
$$

where

$$
F_{k}(t, x):=\varphi(x) f_{k}(t, x)
$$


and $\varphi(x)$ is a $C^{\infty}(\mathbb{R}, I)$ function such that

$$
\varphi(x)= \begin{cases}1, & |x| \leq N_{k} \\ 0, & |x| \geq N_{k}+\varepsilon_{1}\end{cases}
$$

and $0<\varphi(x)<1$ for remaining values of $x$. Then

$$
\max \left\{\left|F_{k}(t, x)\right|: t \in I, x \in R\right\} \leq M_{k}+\varepsilon_{2} .
$$

Notice that both positive $\varepsilon_{1}$ and $\varepsilon_{2}$ can be made arbitrarily small. Denote

$$
\Omega_{k}=\left\{(t, x): 0 \leq t \leq 1, \quad|x(t)| \leq N_{k}\right\}
$$

The original problem (3.1), (3.2) and the quasi-linear one (3.9), (3.2) are equivalent in $\Omega_{k}$.

The quasi-linear problem (3.9), (3.2) can be written in the integral form

$$
x(t)=\int_{0}^{1} G_{k}(t, s) F_{k}(s, x(s)) d s,
$$

where $G_{k}(t, s)$ is the Green function for the respective homogeneous problem. It is given by

$$
G_{k}(t, s)= \begin{cases}\frac{\sin k(s-1) \sin k t}{k \sin k}, & 0 \leq t \leq s \leq 1, \\ \frac{\sin k(t-1) \sin k s}{k \sin k}, & 0 \leq s<t \leq 1\end{cases}
$$

and satisfies the estimate

$$
\left|G_{k}(t, s)\right| \leq \Gamma_{k}=\frac{1}{k|\sin k|}
$$

It follows from (3.10), (3.12) that

$$
|x(t)| \leq \Gamma_{k}\left(M_{k}+\varepsilon_{2}\right) .
$$

If the inequality

$$
\Gamma_{k} M_{k}<N_{k}
$$

holds, and $\varepsilon_{2}$ is such that also $\Gamma_{k}\left(M_{k}+\varepsilon_{2}\right)<N_{k}$ (and this is the case), then a solution $x(t)$ of the quasi-linear problem (3.9), (3.2) satisfies the estimate

$$
|x(t)|<N_{k}, \quad \forall t \in[0,1]
$$

and the original problem (3.1), (3.2) allows for quasilinearization with respect to the domain $\Omega_{k}$ and the linear part $\left(L_{2} x\right)(t):=x^{\prime \prime}+k^{2} x$. It follows from Theorem 1 that if the linear part $L_{2} x$ is $i$-nonresonant, then the problem (3.1), (3.2) has an $i$-type solution. 
Consider the inequality (3.13) and assume that $q(t)$ satisfies the estimates (3.3). If $p>1$, then

$$
\begin{gathered}
\max _{t \in[0,1]} m_{k}(t)=\left(\frac{k^{2}}{p}\right)^{\frac{p}{p-1}}|p-1| q_{1}^{\frac{1}{1-p}}, \\
\min _{t \in[0,1]} n_{k}(t)=\left(\frac{k^{2}}{q_{2}}\right)^{\frac{1}{p-1}} \beta ;
\end{gathered}
$$

but in the case of $0<p<1$ we have

$$
\begin{gathered}
\max _{t \in[0,1]} m_{k}(t)=\left(\frac{k^{2}}{p}\right)^{\frac{p}{p-1}}|p-1| q_{2}^{\frac{1}{1-p}}, \\
\min _{t \in[0,1]} n_{k}(t)=\left(\frac{k^{2}}{q_{1}}\right)^{\frac{1}{p-1}} \beta .
\end{gathered}
$$

Hence the inequality (3.13) reduces to (3.4). The proof is complete.

Corollary 3. If there exist numbers $k_{j} \in\left(i_{j} \pi,\left(i_{j}+1\right) \pi\right), j=1,2, \ldots, n$, which satisfy the inequality (3.4), then there exist at least $n$ solutions (of different types) of the problem (3.1), (3.2).

For $k$ of the form $k=\frac{\pi}{2}+\pi n$, where $n=0,1,2, \ldots$, the basic inequality (3.4) to be verified takes the form

$$
k<\beta \frac{p^{\frac{p}{p-1}}}{|p-1|}\left(\frac{q_{1}}{q_{2}}\right)^{\frac{1}{|p-1|}} .
$$

In Appendix we provide two tables of the results of calculations. The superlinear $(p>1)$ and sublinear $(0<p<1)$ cases are treated separately. For certain values of $p$ and $\frac{q_{1}}{q_{2}}$, the values of $k$ of the form $\frac{\pi}{2}+\pi n, n=0,1,2, \ldots$ are given which satisfy the inequality (3.16).

The number of appropriate $k$ gives the lower bound of the number of solutions of different type to the BVP (3.1), (3.2). For instance, if $p=\frac{2}{3}$ and $\frac{q_{1}}{q_{2}}=\frac{4}{5}$, then there exist at least 2 solutions of different types (namely, a 0-type solution and a 1-type solution). If for the same $p$ the ratio $\frac{q_{1}}{q_{2}}=\frac{16}{17}$, then there exist at least 3 solutions of different types, and so on. 


\section{Appendix}

Table 1. Superlinear case $p>1$.

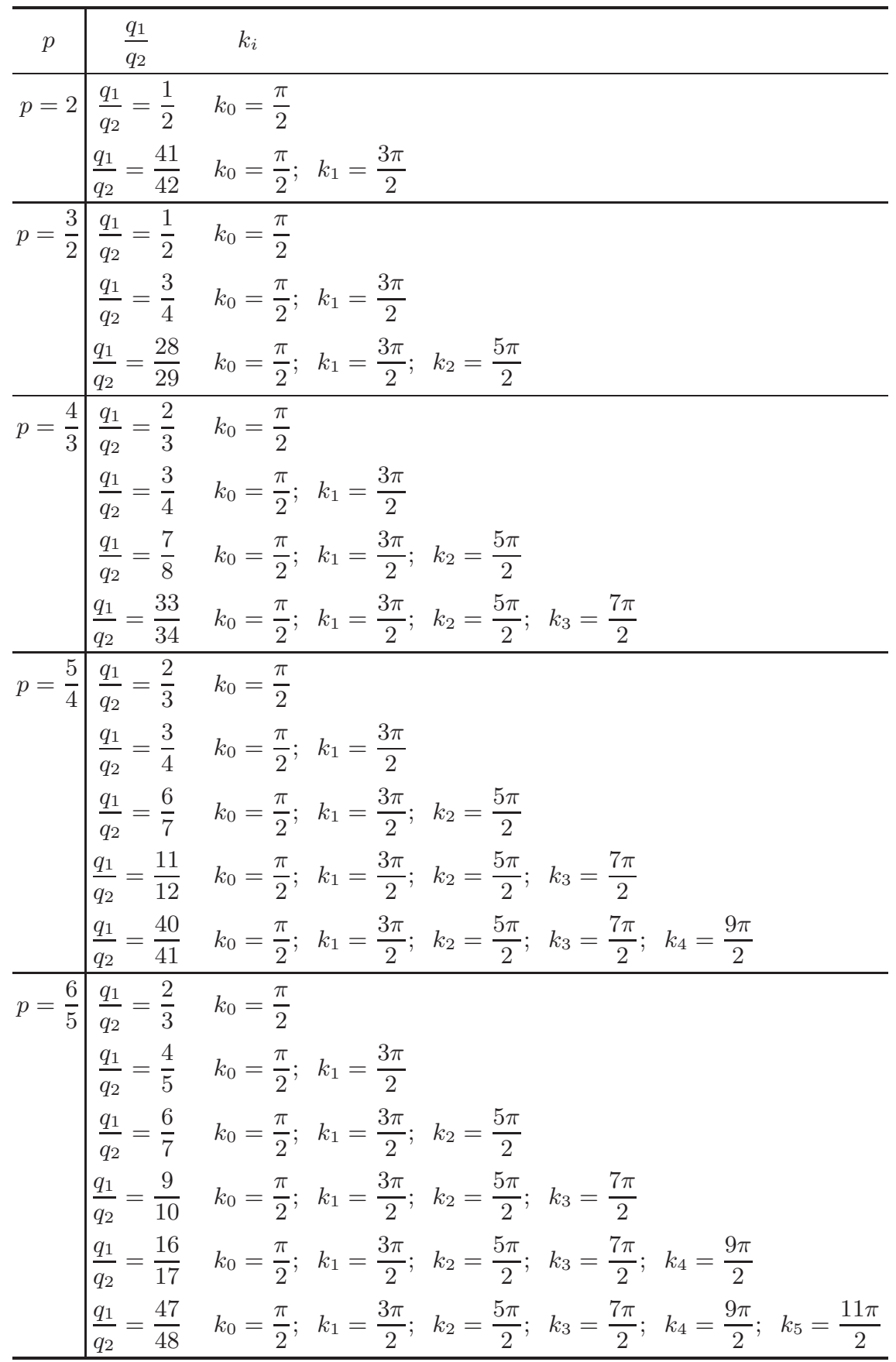


Table 2. Sublinear case $0<p<1$.

\begin{tabular}{c|ll}
\hline$p$ & $\frac{q_{1}}{q_{2}}$ & $k_{i}$ \\
\hline$p=\frac{1}{2}$ & $\frac{q_{1}}{q_{2}}=\frac{2}{3}$ & $k_{0}=\frac{\pi}{2}$ \\
$\frac{q_{1}}{q_{2}}=\frac{9}{10}$ & $k_{0}=\frac{\pi}{2} ; \quad k_{1}=\frac{3 \pi}{2}$ \\
\hline$p=\frac{2}{3}$ & $\frac{q_{1}}{q_{2}}=\frac{2}{3}$ & $k_{0}=\frac{\pi}{2}$ \\
$\frac{q_{1}}{q_{2}}=\frac{4}{5}$ & $k_{0}=\frac{\pi}{2} ; \quad k_{1}=\frac{3 \pi}{2}$ \\
$\frac{q_{1}}{q_{2}}=\frac{16}{17}$ & $k_{0}=\frac{\pi}{2} ; \quad k_{1}=\frac{3 \pi}{2} ; \quad k_{2}=\frac{5 \pi}{2}$ \\
\hline$p=\frac{3}{4}$ & $\frac{q_{1}}{q_{2}}=\frac{2}{3}$ & $k_{0}=\frac{\pi}{2}$ \\
$\frac{q_{1}}{q_{2}}=\frac{4}{5}$ & $k_{0}=\frac{\pi}{2} ; \quad k_{1}=\frac{3 \pi}{2}$ \\
$\frac{q_{1}}{q_{2}}=\frac{8}{9}$ & $k_{0}=\frac{\pi}{2} ; \quad k_{1}=\frac{3 \pi}{2} ; \quad k_{2}=\frac{5 \pi}{2}$ \\
$\frac{q_{1}}{q_{2}}=\frac{23}{24}$ & $k_{0}=\frac{\pi}{2} ; \quad k_{1}=\frac{3 \pi}{2} ; \quad k_{2}=\frac{5 \pi}{2} ; \quad k_{3}=\frac{7 \pi}{2}$ \\
\hline$\frac{q_{1}}{q_{2}}=\frac{2}{3}$ & $k_{0}=\frac{\pi}{2}$ \\
$\frac{q_{1}}{q_{2}}=\frac{4}{5}$ & $k_{0}=\frac{\pi}{2} ; \quad k_{1}=\frac{3 \pi}{2}$ \\
$\frac{q_{1}}{q_{2}}=\frac{7}{8}$ & $k_{0}=\frac{\pi}{2} ; \quad k_{1}=\frac{3 \pi}{2} ; \quad k_{2}=\frac{5 \pi}{2}$ \\
$\frac{q_{1}}{q_{2}}=\frac{12}{13}$ & $k_{0}=\frac{\pi}{2} ; \quad k_{1}=\frac{3 \pi}{2} ; \quad k_{2}=\frac{5 \pi}{2} ; \quad k_{3}=\frac{7 \pi}{2}$ \\
$\frac{q_{1}}{q_{2}}=\frac{31}{32}$ & $k_{0}=\frac{\pi}{2} ; \quad k_{1}=\frac{3 \pi}{2} ; \quad k_{2}=\frac{5 \pi}{2} ; \quad k_{3}=\frac{7 \pi}{2} ; \quad k_{4}=\frac{9 \pi}{2}$ \\
$\frac{q_{1}}{q_{2}}=\frac{2}{q_{2}}$ & $k_{0}=\frac{\pi}{2}$ & $k_{0}=\frac{\pi}{2} ; \quad k_{1}=\frac{3 \pi}{2}$ \\
$\frac{q_{1}}{q_{2}}=\frac{6}{7}$ & $k_{0}=\frac{\pi}{2} ; \quad k_{1}=\frac{3 \pi}{2} ; \quad k_{2}=\frac{5 \pi}{2}$ \\
$\frac{q_{1}}{q_{2}}=\frac{10}{11}$ & $k_{0}=\frac{\pi}{2} ; \quad k_{1}=\frac{3 \pi}{2} ; \quad k_{2}=\frac{5 \pi}{2} ; \quad k_{3}=\frac{7 \pi}{2}$ \\
\hline$q_{1}$
\end{tabular}




\section{References}

[1] S. Bernfeld and V. Lakshmikantham. Introduction to Nonlinear Boundary Value Problems. New York: Academic Press, 1974.

[2] L. H. Erbe. Nonlinear boundary value problems for second order differential equations. J. Differential Equations, 7, 459 - 472, 1970.

[3] L. K. Jackson and K. W. Schrader. Comparison theorems for nonlinear differential equations. J. Differential Equations, 3, 248 - 255, 1967.

[4] F. Sadyrbaev and I. Yermachenko. Types of solutions and multiplicity results for twopoint nonlinear boundary value problems. In: V. Lakshmikantham(Ed.), Fourth World Congress of Nonlinear Analysts WCNA-2004, Orlando, Florida, USA, June 30 - July 7 , 2004, Proceedings. Elsevier, 2004. (accepted for publication)

[5] I. Yermachenko. Multiple solutions of Sturm-Liouville type boundary value problems. In: A. Reinfelds(Ed.), Proc. of the 5th Latvian Mathematical Conference, Daugavpils, Latvia, 2004, Acta Societatis Mathematicae Latviensis, No. 6, DU "Saule", Daugavpils, 61,2004

[6] I. Yermachenko and F. Sadyrbaev. Types of solutions of the second order Neumann problem: multiple solutions. Mathematics. Differential equations (Univ. of Latvia, Institute of Math. and Comp. Sci.), 4, 5 - 21, $2004 . \quad$ (electr. version http://www.lumii.lv/sbornik1/Sbornik-2004-english.htm)

\section{Emdemo-Faulerio tipo lygties kvazelinerizavimas ir daugialypiai sprendiniai}

I, Yermachenko, F. Sadyrbaev

Darbe nagrinejjamas taip vadinamas Emdeno-Faulerio kvazitiesinės diferencialinès lygties homogeninio kraštinio uždavinio sprendinių egzistavimas ir daugialypumas. Parodyta, kad šio uždavinio sprendinio daugialypumas priklauso nuo tam tikru būdu gautos kvazilinearizuotos lygties tiesinès dalies savybių. 